Document downloaded from:

http://hdl.handle.net/10251/65743

This paper must be cited as:

Querol Vives, A.; Gallardo Bermell, S.; Ródenas Diago, J.; Verdú Martín, GJ. (2015). Using lattice tools and unfolding methods for hpge detector efficiency simulation with the Monte Carlo code MCNP5. Radiation Physics and Chemistry. 116:219-225.

doi:10.1016/j.radphyschem.2015.01.027.

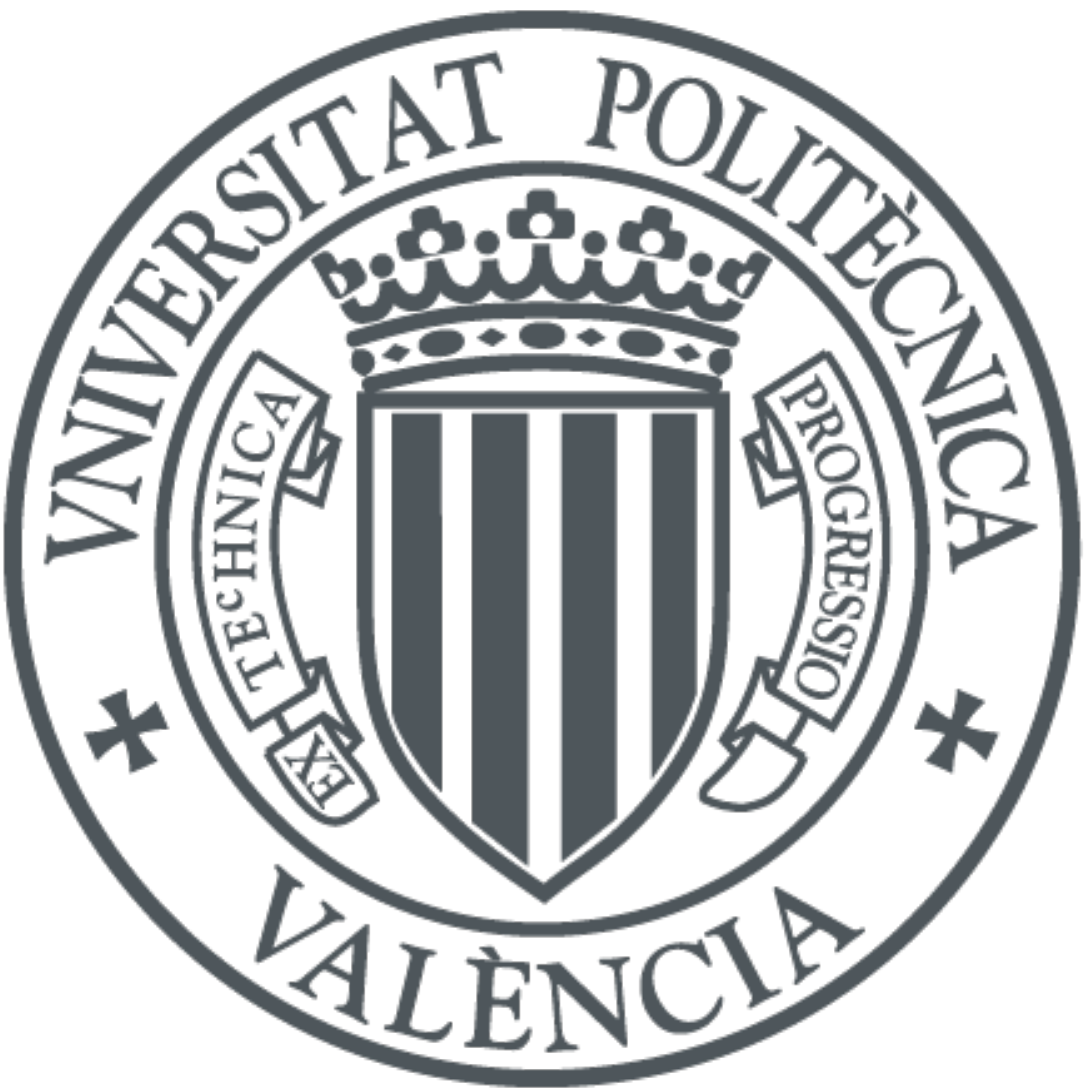

The final publication is available at

http://dx.doi.org/10.1016/j.radphyschem.2015.01.027

Copyright Elsevier

Additional Information 


\title{
USING LATTICE TOOLS AND UNFOLDING METHODS FOR HPGE DETECTOR EFFICIENCY SIMULATION WITH THE MONTE CARLO CODE MCNP5
}

\author{
A. Querol ${ }^{1}$, S. Gallardo, J. Ródenas and G. Verdú \\ Instituto de Seguridad Industrial, Radiofísica y Medioambiental (ISIRYM) \\ Universitat Politècnica de València, Valencia, Spain.
}

\begin{abstract}
In environmental radioactivity measurements, High Purity Germanium (HPGe) detectors are commonly used due to their excellent resolution. Efficiency calibration of detectors is essential to determine activity of radionuclides. The Monte Carlo method has been proved to be a powerful tool to complement efficiency calculations. In aged detectors, efficiency is partially deteriorated due to the dead layer increasing and consequently, the active volume decreasing. The characterization of the radiation transport in the dead layer is essential for a realistic HPGe simulation. In this work, the MCNP5 code is used to calculate the detector efficiency. The F4MESH tally is used to determine the photon and electron fluence in the dead layer and the active volume. The energy deposited in the Ge has been analyzed using the *F8 tally. The F8 tally is used to obtain spectra and to calculate the detector efficiency. When the photon fluence and the energy deposition in the crystal are known, some unfolding methods can be used to estimate the activity of a given source. In this way, the efficiency is obtained and serves to verify the value obtained by other methods.
\end{abstract}

Key Words: HPGe detector, dead layer, Monte Carlo, lattice tools

\section{INTRODUCTION}

Gamma spectrometry is of great importance for determining radionuclide activities. It does not require chemical separation and can be measured in bulk form (Conti et al., 2013, Santo et al., 2012). HPGe detectors are widely used for gamma spectrometry, mainly because of the high sensitivity and energy resolution, especially for low-level activity materials. However, the experimental determination of the detector efficiency presents some difficulties. It requires the availability of standard sources of a mix of radionuclides in the same counting geometry that the samples will be measured. Another way to determine efficiency is using the Monte Carlo method, which is widely used for simulation of HPGe detectors. It is a very flexible and powerful tool, which enables the analysis of a wide energy range. It allows optimizing time and financial resources. MCNP5, GEANT4 and PENELOPE codes, based on the Monte Carlo method, are a reliable tool for this purpose. Manufacturers provide usually detailed information about geometry and resolution, including sometimes features related to dead layer and electric contacts. However, in aged detectors, detector efficiency is deteriorated partially due to the dead layer thickness increasing and consequently the decreasing of the active

\footnotetext{
${ }^{1}$ Corresponding author: Andrea Querol. Phone number: +34 963879631; Fax: +34 963877634; email: anquevi@upv.es
} 
detection volume (Huy et al., 2007). Thus, some significant discrepancies between simulated and experimental efficiencies have been reported in the literature (Ródenas et al., 2007; Huy, 2010). For this reason, the characterization of the radiation transport in the dead layer is essential for a realistic HPGe simulation.

In this frame, an HPGe coaxial detector has been simulated using the MCNP5 code (X-5 Monte Carlo Team, 2003). To determine both photon and electron fluence throughout different layers of the Ge crystal a lattice tool (F4MESH tally) has been used. It consists of a superimposed mesh over all the Ge crystal and allows determining the penetration of photons as well as the electron production in the inactive and active volume of the crystal. The energy deposited in the Ge crystal has been analyzed using the *F8 tally $(\mathrm{MeV})$ for different dead layer thicknesses. The F8 tally, which records the Pulse Height Distribution (PHD), is used to obtain spectra and to calculate the detector efficiency. These results allow estimating the actual dead layer thickness value for the HPGe detector and to compare it with the value provided by the manufacturer. Afterwards, unfolding methods can be used to obtain the detector efficiency from unfolded spectra, using the response matrix of the system. This matrix defines the relation between PHD and the spectrum and it can be obtained by simulating several monochromatic beams.

\section{MATERIALS AND METHODS}

\subsection{Experimental measurements}

An HPGe coaxial detector system has been used for experimental measurements in the Laboratorio de Radiactividad Ambiental (LRA) of the Universitat Politècnica de València (UPV). The detector model is ORTECGMX40P4, with a closed-end coaxial geometry. The main specifications of the detector are the following: $40 \%$ relative efficiency at $1.33 \mathrm{MeV}$ Co-60; FWHM resolution of $2.0 \mathrm{keV}$; peak shape FWTM/FWHM $2.0 \mathrm{keV}$ at $1.33 \mathrm{MeV} \mathrm{Co}-60$; and the peak-to-Compton ratio 59 for Co- 60 . The crystal diameter is $60 \mathrm{~mm}$ and the length is $71.1 \mathrm{~mm}$. The core hole diameter is $9 \mathrm{~mm}$, and the core hole length is $63.1 \mathrm{~mm}$. The end cap to crystal distance is $4 \mathrm{~mm}$. The cup length is $105 \mathrm{~mm}$. The cryostat window material is aluminum with $0.8 \mathrm{~mm}$ thickness. The crystal has also a layer $0.03 \mathrm{~mm}$ of Mylar in front of the cryostat window. The effective thickness of the dead layer is not well known due to the existence of a transition zone between the inactive layer and the active germanium in the crystal whose thickness is very difficult to be accurately estimated. The manufacturer provides a value of $700 \mu \mathrm{m} \mathrm{Ge} / \mathrm{Li}$ dead layer in the crystal inner hole and $0.3 \mu \mathrm{m} \mathrm{Ge} / \mathrm{B}$ dead layer in the top and lateral crystal sides. A $100 \mathrm{cc}$ Petri dish made of polyethylene filled with water containing a gamma standard solution is used. The source used for measurements is a calibration gamma standard solution, covering the energy range between 59 and $1840 \mathrm{keV}$. The radionuclides contained in the source solution are listed in Table 1 together with their main peak energy, branching ratio and certified activity. Figure 1 shows the geometry of the HPGe detector and the MCNP5 model. 


\subsection{HPGe MCNP5 model}

The code has been used for simulating the experimental detection device of LRA. The dead layer thickness is usually obtained after an iterative process comparing experimental versus simulated results varying the thickness. The simulation process is repeated, adjusting the dead layer thickness, until results show a good agreement with experimental data. Two MCNP5 models have been developed to estimate the dead layer thickness: 1) a simplified model with a point source and just the Ge crystal, and 2) a model with a Petri dish source and the full HPGe detector reproducing the main features provided by the manufacturer. The Petri dish is situated on top of the detector providing a relatively highefficiency geometry. Figure 2 shows the geometry of both models considered.

Twenty million particles have been simulated to obtain statistical errors lower than 1.0 $\%$. MODE P, E has been activated to follow tracks of photons and electrons. The lattice F4MESH has been used to calculate the photons and electrons fluence $\left(1 / \mathrm{cm}^{2}\right)$ in a superimposed mesh tally over the Ge crystal. The *F8 tally has been used to obtain the energy deposited (MeV) in ten cells of $10 \mu \mathrm{m}$ considered in the surface of the Ge crystal. Moreover, the PHD in the detector has been recorded using the F8 tally. It has been measured in 8192 channels corresponding to the number of channels of the actual detector device. All MCNP5 results are normalized per photon emitted by the source.

\section{RESULTS AND DISCUSSION}

Main results obtained simulating the HPGe detector with the MCNP5 code are presented. Different MCNP5 records have been considered: a lattice tool (F4MESH tally) to determine the photon and electron fluence, an *F8 tally to measure the energy deposited in the Ge crystal and an F8 tally to obtain the spectrum and to calculate the detector efficiency. With these results, the actual dead layer thickness value of the HPGe detector is determined. Once this value is known, unfolding methods can be used to obtain the detector efficiency from unfolded spectra.

\subsection{F4MESH Tally}

Using F4MESH, the photon fluence is obtained in a fine mesh over the Ge crystal. Results corresponding to Am-241, Cd-109, Cs-137 and Zn-65 are shown in Figure 3.

For Am-241 and Cd-109, due to the low photon energy, most of interactions occur along a very small distance from the crystal surface, lower than $0.2 \mathrm{~cm}$. It justifies the important dead layer effect in the efficiency detection of these radionuclides. For this reason, F4MESH has been obtained in $1 \mathrm{~cm}$ of Ge crystal divided in 1000 cells. However, for Cs137 and $\mathrm{Zn}-65$, interactions occur at a higher depth because they emit more penetrating gamma photons. In these cases, the detection efficiency is not influenced by the dead layer thickness.

As low energy photons mainly interact closer to the crystal surface, some of the secondary electrons may deposit part of their energies in the dead layer and are not collected. To take into account this process, electrons and photons must be considered in the simulation. Results obtained show that the electron fluence per photon emitted for Am-241 is reduced from $2.5 \cdot 10^{-4}$ to $0.1 \cdot 10^{-4}$ in less of $2 \mathrm{~mm}$. For Cd-109, the electron fluence per photon 
emitted is reduced from $1.8 \cdot 10^{-4}$ to $0.2 \cdot 10^{-4}$ in similar Ge length. However, for Cs- 137 and $\mathrm{Zn}-65$ the Compton and photoelectric electrons are produced along all the Ge crystal.

\subsection{F8 and *F8 tallies}

The *F8 tally (MeV) has been used in both MCNP5 models to obtain the energy deposition in the dead layer varying its thickness. Results obtained are shown in Figure 4. As it can be seen, for both models a lineal relation between dead layer thickness and absorbed energy for each radionuclide is observed. The *F8 tally calculated for Am-241 is about twice the value obtained for Cd-109. With the detailed model, the deposited energy is lower than in the simplified model, as expected a cause of the greater number of layers attenuating the radiation.

The spectrum have been obtained from the PHD using the F8 tally, recorded in the entire Ge crystal. Figure 5 shows different spectra obtained using both models. X rays from Ge appears in all cases at energies lower than the photopeak when the simplified model is used. However, for the detailed model, they do not appear in the spectrum and are subtracted from the photopeak. Furthermore, other differences between results obtained with each model can be observed at both the Bremsstrahlung zone and the Compton border.

Figure 6 shows the efficiency obtained with F8 tally considering different dead layer thicknesses and both MCNP5 models. Efficiency obtained with the simplified model is lower than using the detailed model. In both cases, results stated that the dead layer is not important for Cs-137 and Zn-65. Nevertheless, using the point source model the efficiency for Am-241 in $100 \mu \mathrm{m}$ varies from 0.45 to 0.35 (22\%), while for Cd-109 does from 0.45 to $0.41(9 \%)$, and for Co-57 from 0.44 to $0.41(7 \%)$. With the detailed model the efficiency variation for Am-241 in $100 \mu \mathrm{m}$ is just from 0.14 to $0.115(8 \%)$ and for Cd-109 and Co-57 is very low. In general, the simplified model is more efficient than the detailed model. But, it is a simplification.

The actual dead layer thickness of the HPGe detector used in the LRA of the UPV has been obtained applying the detailed model. Simulated and experimental efficiencies obtained for a gamma standard source are compared in Table 2. From these values, an average dead layer thickness between 70 and $80 \mu \mathrm{m}$ is obtained.

Once the actual dead layer of the HPGe detector is known, the efficiency curve can be obtained with the MCNP5 code using a response matrix of the system and applying unfolding methods.

\subsection{Unfolding spectra to obtain the detector efficiency}

The relation between PHD and the spectrum is defined by a response function, usually expressed by means of a matrix, $\mathrm{R}$ :

$$
R \cdot \vec{s}=\vec{m}
$$

where $\vec{s}$ is the unknown spectrum and $\vec{m}$ is the PHD recorded in the HPGe detector for a gamma standard solution (Table 1) covering the energy range between 59 and $1840 \mathrm{keV}$.

The response matrix can be obtained recording the PHD (F8 tally) produced by different monochromatic beams from 1 to $1840 \mathrm{keV}$, with an energy beam of $4 \mathrm{keV}$ (460 x 460 
matrix dimensions) with the MNCP5 model developed. Figure 7 shows the response matrix obtained.

Once the whole response matrix is obtained, the unknown spectrum could be estimated from Eq. 1. However, obtaining $\mathrm{R}^{-1}$ is very difficult with a standard numerical procedure because this matrix is ill-conditioned and rank deficient, so there are a large number of solutions for the Least Squares problem. Since $\mathrm{R}$ is a real $\mathrm{M} \times \mathrm{N}$ matrix, it admits a Singular Value Decomposition (SVD) (Golub and Van Loan, 1996).

An optimal solution can be obtained generating a new response matrix and removing the parts of the solution corresponding to the smallest singular values. The Truncated SVD (TSVD) method (Hansen, 1994) can be used to obtain a new matrix, $\mathrm{R}_{\mathrm{k}}$, where $\mathrm{k}$ is the regularization parameter. It is the number of singular values of $\mathrm{R}$ considered. TSVD regularization method is based on this observation and solves the problem:

$$
\min \|\overrightarrow{\tilde{s}}\|_{2} \text { subject to } \min \left\|R_{k} \overrightarrow{\tilde{s}}-\vec{m}\right\|_{2}
$$

The quality of the TSVD results strongly depends on the regularization parameter. For this reason, a reliable method for determination of this parameter is crucial to solve the inverse problem. There are some powerful techniques to estimate the optimal parameter. In this work, it has been selected plotting the L-curve (Hansen, 1994, 1998), which consists in representing the 2-norm of the solution vector versus the 2-norm of the residual vector. The optimal parameter corresponds with the corner of the L-curve because this value minimizes the solution and residual norms at the same time.

Once the optimal value of $\mathrm{k}$ is known, the TSVD method can be applied to obtain the unfolded spectrum. The unfolded efficiency can be calculated from the relation between the recorded PHD and the unfolded spectrum. Furthermore, the detector efficiency can be obtained from the recorded PHD. Figure 8 shows the unfolded efficiency in comparison to the efficiency obtained from the PHD recorded and the experimental efficiency. As it can be seen, good results have been obtained.

\section{CONCLUSIONS}

Using lattice tools of the MCNP5 code to estimate the actual dead layer thickness of an HPGe detector is a good method to characterize the device for environmental measurements.

F4MESH, F8 and *F8 tallies contribute to determine the real interaction zone of photons and electrons in the crystal using the simplified model. However, this model is an approximation and the detailed model that better represents the actual process is applied for further calculations to obtain the real dead layer thickness (between 70 and $80 \mu \mathrm{m}$ ).

Unfolding methods such as the TSVD together with the MC method permit to obtain the detector efficiency with an acceptable accuracy. However, some discrepancies appear at higher energies due to the summing effect of the radionuclides used. 


\section{REFERENCES}

Conti, C. C., Salinas,I. C. P., Zylberberg, H., 2013. A detailed procedure to simulate an HPGe detector with MCNP5, Progress in Nuclear Energy, 66, pp. 35-40.

Golub, G.H., Van Loan C.F., 1996. Matrix Computations, Third edition The Johns Hopkins University Press.

Hansen, P.C., 1994. Regularization tools: A Matlab package for analysis and solution of discrete ill-posed problems, Numerical Algorithms, 6, pp.1-35.

Hansen, P.C., 1998. Rank-Deficient and Discrete Ill-Posed Problems, SIAM, Philadelphia.

Huy, N. Q., Binh, D. Q., An, V. X., 2007. An, Study on the increase of inactive germanium layer in a high-purity germanium detector after a long time operation applying MCNP code, Nuclear Instruments and Methods in Physics Research, 573, pp. 384-388.

Huy, N. Q., 2010. The influence of dead layer thickness increase on efficiency decrease for a coaxial HPGe p-type detector, Nuclear Instruments and Methods in Physics Research A, 621, pp. 390-394.

Ródenas, J., Gallardo, S., Ballester, S., Primault, V., Ortiz, J., 2007. Application of the Monte Carlo method to the analysis of measurement geometries for the calibration of a HPGe detector in an environmental radioactivity laboratory, Nuclear Instruments and Methods in Physics Research B, 263, pp.144-148.

Santo, A.S.E., Wasserman, F. G., Conti, C. C., 2012. HPGe well detector calibration procedure by MCNP5 Monte Carlo computer code, Annals of Nuclear Energy, 46, pp. 213-217.

X-5 MONTE CARLO TEAM, "MCNP - A General Monte Carlo N particle Transport Code, Version 5" LA-UR-03-1987, Los Alamos National Laboratory, April, 2003. 
Table 1. Gamma standard solution features.

Table 2. Experimental and simulated efficiency for different dead layer thicknesses. 
Figure 1. Geometry of the HPGe detector and MCNP5 model.

Figure 2. MCNP5 models: a) simplified model and b) HPGe detailed model

Figure 3. F4MESH photons and electrons fluence $(1 / \mathrm{cm} 2)$ for four radionuclides

Figure 4. *F8 for different dead layer thicknesses and both the point-source and Petridish geometries

Figure 5. Pulse height distributions for four radionuclides and both the point-source and Petri-dish geometries

Figure 6. Efficiency for different dead layer thicknesses and both the point-source and Petri-dish geometries

Figure 7. Response matrix

Figure 8. Unfolded efficiency 